\title{
Sessile Serrated Polyps: An Important Route to Colorectal Cancer
}

Matthew F. Kalady, MD

\begin{abstract}
The serrated neoplastic pathway accounts for approximately $30 \%$ of colorectal cancers. Characterized by mutations in the oncogene $B R A F$, DNA promoter hypermethylation, and microsatellite instability, cancers arising in this pathway develop via serrated polyp intermediates. Serrated polyps represent a heterogeneous group of lesions with distinct genetic, molecular, and clinical features. Sessile serrated polyps (also called sessile serrated adenomas) have emerged as the key intermediates in this pathway. These lesions have malignant potential and are often difficult to detect endoscopically, thus contributing to the development of interval cancers. Recent advances in the understanding of sessile serrated polyps have led to new histologic classifications, increased endoscopic recognition, and changes in clinical management recommendations. This article focuses on sessile serrated polyps as a unique and important route to colorectal cancer. (JNCCN 2013;11:1585-1594)
\end{abstract}

\section{Classification of Serrated Polyps}

Traditionally, colorectal polyps were broadly classified into adenomatous (neoplastic with malignant potential) and non-neoplastic (without malignant potential), which included hyperplastic and hamartomatous polyps. In the early 1990s, hyperplastic polyps began to be recognized as a diverse group of lesions, each with distinct pathologic features. Histologic subsets began to be defined within the general category of what is now called serrated polyps. Serrated polyps are characterized

From the Department of Colorectal Surgery, Digestive Disease Institute, Cleveland Clinic, Cleveland, Ohio.

Submitted July 25, 2013; accepted for publication November 8, 2013.

Dr. Kalady has disclosed that he has no financial interests, arrangements, affiliations, or commercial interests with the manufacturers of any products discussed in this article or their competitors. Dr. Kalady is the Krause-Lieberman Chair in Colorectal Surgery.

Correspondence: Matthew F. Kalady, MD, Cleveland Clinic, 9500 Euclid Avenue, A30, Cleveland, OH 44195. E-mail: kaladym@ccf.org by a saw-tooth or serrated glandular pattern that is hypothesized to occur because of the lack of apoptosis of dividing cells within the colonic crypt. As these cells proliferate without apoptotic clearing, they fold onto each other, and this infolding produces the classic serrated appearance. Although these relatively newly recognized lesions have had many nomenclature iterations, the WHO has provided definitions of serrated polyps to provide a more consistent recognition, with classification into 3 major groupings: hyperplastic polyps (HPs), sessile serrated adenomas (SSAs) or sessile serrated polyps (SSPs), and traditional serrated adenomas (TSAs). ${ }^{1}$ The terms SSA and SSP are synonymous, and experts have opted to use sessile serrated adenoma/polyp (SSA/P) to avoid confusion between the terms. ${ }^{2}$ This convention will be used throughout this article.

HPs are characterized by straight, symmetrical crypts that tend to have a wide crypt opening relative to the base, because the proliferation and serrations predominate at the top of the crypt (Figure 1A). HPs are further subdivided by WHO classification into microvesicular, goblet cell, and mucin-poor. Microvesicular HPs are believed to be an initial lesion in the serrated pathway to cancer. HPs are the most common serrated polyp and are commonly located in the distal colon and rectum.

Longacre and Fenoglio-Preiser ${ }^{3}$ first recognized a subset of hyperplastic polyps with dysplasia as a distinct form of colorectal neoplasia. The concept of this atypical variant of nondysplastic hyperplastic polyp (SSA/P) was further developed and elucidated by Torlakovic et al. ${ }^{4,5} \mathrm{SSA} / \mathrm{Ps}$ demonstrate a distorted and disorganized crypt growth. Although proliferating cells may be seen anywhere along the crypt axis, the base of the crypt often contains a hyperproliferative zone that results in asymmetrical serrations and oddly shaped structures at the base (Figure 1B). The crypts may appear dilated or branched, and thus have been referred to as L-shaped, 


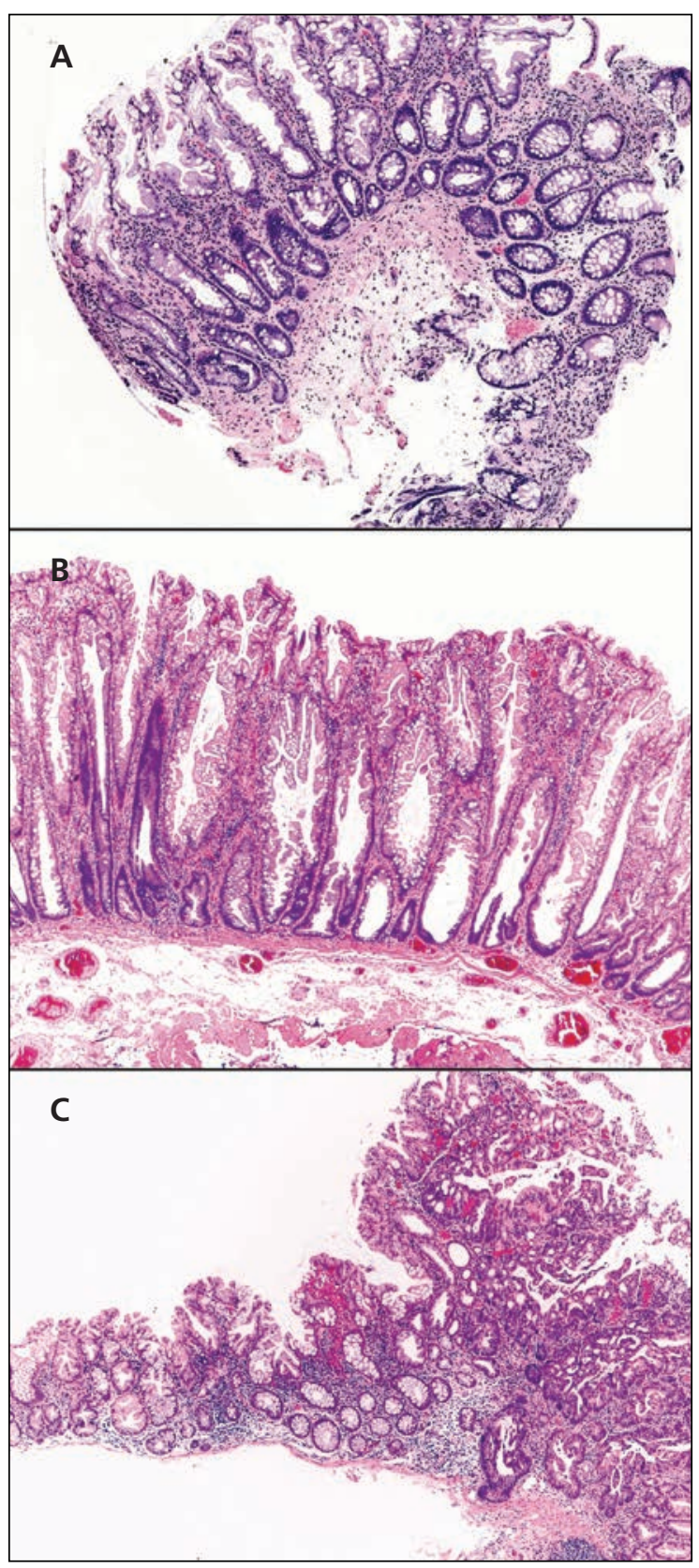

Figure 1 (A) Hyperplastic polyps are characterized by elongated, straight crypts with luminal serrations that are limited to the upper two-thirds of the crypt (hematoxylineosin [H\&E], original magnification $x 40$ ). The crypt bases are uniform and there is no cytologic dysplasia. (B) In sessile serrated adenomas/polyps, the crypts are often dilated and tend to grow laterally along the muscularis mucosa (H\&E, original magnification $\mathrm{x} 40$ ). Serrations also extend along the entire length of the crypts. (C) Sessile serrated adenomas/ polyps can develop cytologic dysplasia, as illustrated in this example (H\&E, original magnification $\times 20)$. The right portion of this sessile serrated polyp contains cytologic dysplastic epithelium characterized by enlarged and hyperchromatic nuclei. Prominent architectural complexity characterized by cribiforming is also present.

Courtesy of Rish Pai, MD, PhD, Cleveland, $\mathrm{OH}$.
T-shaped, or boot-like. Mucin and goblet cells are also often overrepresented at the base of the crypts. ${ }^{6} \mathrm{~A}$ retrospective review of large lesions that were previously called HPs shows that most of these polyps would now be called SSA/Ps according to the new nomenclature. ${ }^{7} \mathrm{HPs}$ and SSA/Ps are often difficult to distinguish, and there is considerable interobserver variability among pathologists. ${ }^{8,9}$

$\mathrm{SSA} / \mathrm{Ps}$ may be further classified according to the presence of dysplasia (Figure 1C). SSA/Ps may have a focus of conventional adenoma-like dysplasia, which represents malignant progression. ${ }^{1,2}$ These lesions were previously called mixed polyps or collision polyps because they were thought to have been a coexistence of 2 separate entities, rather than a progression of disease, as is now supported. Conventional adenomatous dysplastic areas in SSA/Ps have a similar cytologic appearance to classic tubular or tubulovillous adenomas. They have elongated cells with increased mitoses, hyperchromatic pseudostratified nuclei, and amphophilic cytoplasm. ${ }^{2}$ Expert opinion suggests that SSA/Ps with cytologic dysplasia be considered to have at least the same colorectal cancer (CRC) risk as adenomas with high-grade dysplasia. ${ }^{2}$ Another form of dysplasia can be seen in SSA/Ps, termed serrated dysplasia, which is characterized by proliferation of atypical cuboidal cells with increased mitoses and enlarged, prominent round nucleoli. Although this concept is still not completely understood, some believe that this type of dysplasia represents advanced neoplasia and progression toward malignancy. ${ }^{1}$

TSAs have a more extensive proliferative zone at the base of the crypt and into the surrounding lateral compartment. These are often perpendicular to the long axis of the adenomatous portion. The most characteristic feature of TSAs is the loss of anchorage of the normal crypts to the muscularis mucosa. They have distinct adenomatous portions and malignant potential. TSAs are rare lesions and account for only $1 \%$ of all colon polyps and $2 \%$ of all serrated lesions. ${ }^{1,2}$

\section{Genetic and Molecular Characteristics of the Serrated Pathway to CRC}

CRC has a molecular and genetic heterogeneity that had not been appreciated until the past 2 decades. ${ }^{10}$ Most CRCs arise via the classical adenomato-carcinoma sequence, as proposed by Vogelstein et 
al. ${ }^{11}$ However, approximately $25 \%$ to $30 \%$ of CRCs develop via a pathway characterized by defective DNA mismatch repair (MMR) mechanisms. ${ }^{12} \mathrm{Al}$ though approximately $15 \%$ of MMR-deficient CRCs are caused by a heritable germline mutation, as in Lynch syndrome, the other $85 \%$ arise sporadically secondary to loss of the MMR gene hMLH1 caused by hypermethylation of the gene promoter region, which silences its transcription. The result is a microsatellite unstable or microsatellite instability high (MSI-H) CRC. Increased hypermethylation particularly occurs in promoter areas rich in cytosine and guanine dinucleotide repeats, or $\mathrm{CpG}$ islands. Thus, the associated cancers have been termed CpG island methylator phenotype (CIMP). CIMP CRCs are also associated with mutations in the BRAF oncogene. ${ }^{10,13}$ Current understanding of molecular subtypes of CRC supports that CIMP cancers develop via the serrated pathway to neoplasia, with serrated polyps as the intermediary lesions.

The sequence of events is still not completely characterized, but the belief is that a BRAF mutation is the initiating event, with conversion of normal mucosa to either a microvesicular hyperplastic polyp or an SSA/P. DNA hypermethylation (CIMP) of the promoter region of the mismatch repair gene MLH1 results in lack of MLH1 protein expression, and subsequently microsatellite instability. This eventually leads to cytologic dysplasia, and ultimately to colorectal adenocarcinoma (Figure 2). Genetic and molecular evidence supports SSA/Ps as the intermediary lesions. BRAF mutations (V600E) are found in microvesicular HPs, and more commonly in $\mathrm{SSA} / \mathrm{Ps}(75 \%-82 \%)$, which is similar to that reported for MSI-H colon cancers. ${ }^{10,13,14}$ Furthermore, advanced serrated polyps often exhibit CIMP and loss of MLH1 expression. ${ }^{15,16}$ hMLH1 methylation has been detected in $36 \%$ of HPs, $70 \%$ of SSA/Ps, and $86 \%$ to $87 \%$ of sporadic MSI-H CRCs. ${ }^{14,17,18}$ The rate of progression through this pathway is variable, but it is believed that once microsatellite instability develops, a more rapid progression to cancer occurs. ${ }^{2}$ A recent review provides more detail regarding the genetic changes in serrated lesions. ${ }^{19}$

Evidence also suggests an alternate serrated pathway involving KRAS mutations and microsatellite low or stable and CIMP-low CRCs. This pathway has also been associated with hypermethylation of the DNA repair gene, O-6-methylguanine-DNA

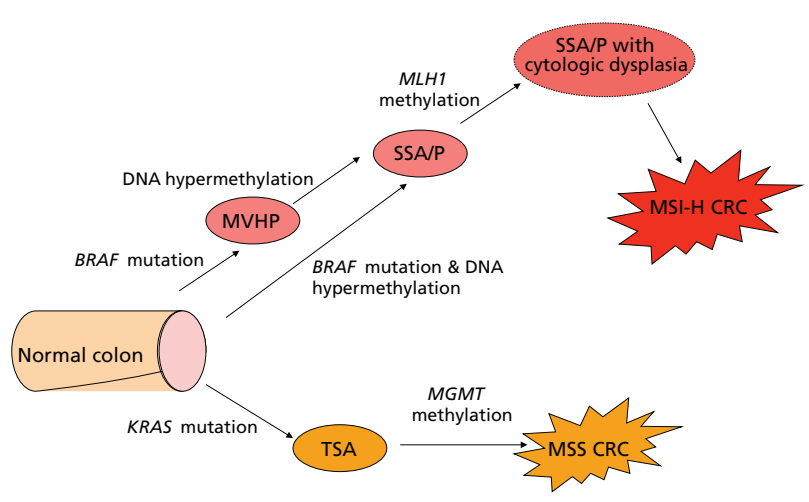

Figure 2 Schematic of the serrated pathway to colorectal neoplasia. Normal colorectal mucosa is transformed into a microvesicular hyperplastic polyp (MVHP) or sessile serrated adenoma/polyp (SSA/P) after an initiating BRAF mutation. DNA hypermethylation leads to CIMP and eventual loss of $M L H 1$ via methylation, which in turn induces dysplasia and eventual progression to microsatellite instability high (MSI-H) colorectal cancer (CRC). Serrated lesions may also develop through a different set of genetic and molecular events characterized by initiating KRAS mutations and methylation of methyl guanine methyltransferase (MGMT), resulting in traditional serrated adenomas (TSAs) as the intermediate lesions and subsequent microsatellite stable (MSS) CRCS.

methyltransferase (MGMT), and TSAs. ${ }^{20,21}$ KRAS mutations are commonly seen in small hyperplastic left-sided polyps and TSAs, but are extremely rare in SSA/Ps. ${ }^{13,22,23}$

\section{Prevalence of SSA/Ps}

As the definition and recognition of SSA/Ps has changed over time, the true prevalence of these lesions is difficult to define. Previously classified as benign hyperplastic polyps, lesions that are now called SSA/Ps were often ignored by endoscopists. Without biopsy or polypectomy to document these lesions, the prevalence of SSA/Ps has been largely underestimated and undefined. Earlier autopsy studies report that $13 \%$ to $40 \%$ of people had colorectal serrated polyps without differentiation between the various, now histologically defined serrated lesions. ${ }^{24,25}$ More recently, Hetzel et $\mathrm{al}^{26}$ reported an SSA/P prevalence of $1.2 \%$ on review of 7192 average-risk screening colonoscopies. Similarly, in a review from the Cleveland Clinic, an SSA/P was identified in $2.1 \%$ of 28,054 colonoscopies. ${ }^{7}$ In one recent study of screening colonoscopies, SSA/Ps were found in as many as $20 \%$ of patients. ${ }^{27}$ Approximately $1 \%$ to $9 \%$ of all colon polyps are SSA/Ps, and between 5\% and $25 \%$ of all serrated lesions are SSA/Ps. ${ }^{26,28-30}$ 


\section{SSA/P Clinical Significance}

Colorectal SSA/Ps are clinically relevant lesions as both markers for other colorectal neoplasia and as polyps with inherent potential for malignant transformation.

\section{Association With Adenomas and Cancer}

Compared with patients without SSA/Ps, those with SSA/Ps have a higher incidence of both synchronous and metachronous polyps, including $\mathrm{HPs},{ }^{26}$ other SSA/Ps, and adenomas. ${ }^{31-33}$ In particular, larger SSA/Ps located in the right colon are associated with an increased risk of synchronous and advanced adenomas. The presence of a proximal SSA/P of $10 \mathrm{~mm}$ or greater is associated with a 2 to 5 relative risk for advanced neoplasia. ${ }^{34}$ Patients with both SSA/Ps and adenomas, as opposed to those with either SSA/Ps or adenomas alone, tend to have larger and more SSA/Ps, and more advanced SSA/Ps and adenomas. ${ }^{35}$ Looking at this from a different perspective, patients with advanced neoplasms are 3.7 times more likely to have synchronous high-risk serrated polyps than those without advanced neoplasms. ${ }^{36}$

In addition to adenomas, SSA/Ps are also associated with both synchronous and metachronous CRCs. In a study from Japan including 10,199 people without previous colonoscopy, the finding of serrated polyps was the strongest predictor of synchronous CRC. ${ }^{37}$ Again, the presence of large right-sided $\mathrm{SSA} / \mathrm{Ps}$ was associated with synchronous CRCs. ${ }^{34}$ Compared with patients with HPs or adenomas alone, SSA/Ps are also associated with an increased risk of metachronous CRC. In a small study of 55 patients who had SSA/Ps, 12.5\% developed metachronous CRC during a follow-up of 7.2 years. This percentage was significantly higher than among matched controls for patients with either HPs $(1.8 \%)$ or adenomas $(1.8 \%))^{32}$ These data underscore the importance of heightened awareness for other colorectal lesions when an SSA/P is identified.

\section{Malignant Potential of SSA/Ps}

Several lines of evidence support the malignant potential of SSA/Ps. Molecular markers for the serrated pathway to cancer have been identified in the precursor polyps. Additional molecular evidence is provided by study of MSI-H CRCs. MSI-H CRCs are more likely to have associated serrated polyps than microsatellite stable cancers. ${ }^{15,38}$ Goldstein et $\mathrm{al}^{38}$ found that patients with previously removed SSA/Ps developed
MSI-H colon cancers in the same anatomic location from which the initial polyp was removed. Conversely, patients with HPs removed in the same locations did not develop cancer.

Histologic elements of SSA/P, dysplasia, and adenocarcinoma can be seen in the same lesion (Figure 3). Lash et $\mathrm{a}^{129}$ reported a series of 2416 SSA/ Ps in which $14 \%$ contained cytologic dysplasia and $1 \%$ had associated adenocarcinoma. Malignant progression in these lesions is believed to contribute to the development of interval cancers. Interval cancers are those that develop within the timeframe of the next recommended colonoscopy evaluation. These instances may represent missed lesions, incomplete removal, or rapid development of new lesions. Interval cancers are 4 times more likely to be MSI$\mathrm{H}$ and to have a methylator phenotype, which are both traits associated with the serrated pathway to neoplasia. ${ }^{17,39}$ Factors associated with SSA/Ps, such as right-sidedness and flat lesions, also make them more difficult to detect endoscopically and may contribute to the development of these types of interval cancers.

The main clinical question for surveillance is how rapidly do these lesions progress toward cancer? Because of the lack of large randomized prospective studies, understanding of the natural history and malignant potential of SSA/Ps remains inconclusive. One small retrospective study reported that the median interval from progression of SSA/P to adenocarcinoma was 7.3 years; $18 \%$ of cases developed within 3 years, and $27 \%$ of cancers developed between 3 and 6 years. ${ }^{38}$ For larger polyps, the interval between SSA/P resection and cancer diagnosis was shorter than for small polyps, suggesting that either larger polyps tend to progress more rapidly or that perhaps that the lesions were incompletely removed. In a retrospective review of successive colonoscopies, Lazarus et $\mathrm{al}^{40}$ reported that SSA/Ps grew at an estimated rate of $3.8 \mathrm{~mm} / \mathrm{y}$ for $\mathrm{SSA} / \mathrm{Ps}$, which was more rapid than the growth rate determined for adenomas $(2.8 \mathrm{~mm} / \mathrm{y})$ and HPs $(1.4 \mathrm{~mm} / \mathrm{y})$. In contrast, epidemiologic studies suggest a slower transition from $\mathrm{SSA} / \mathrm{P}$ to dysplasia and cancer. In reviewing more than $2400 \mathrm{SSA} / \mathrm{Ps}$, the average age at diagnosis for SSA/Ps was 61 years, versus 66 years for detection of SSA/Ps with low-grade dysplasia, 72 years for highgrade dysplasia, and 76 years for adenocarcinoma. Thus, according to this information, the interval 


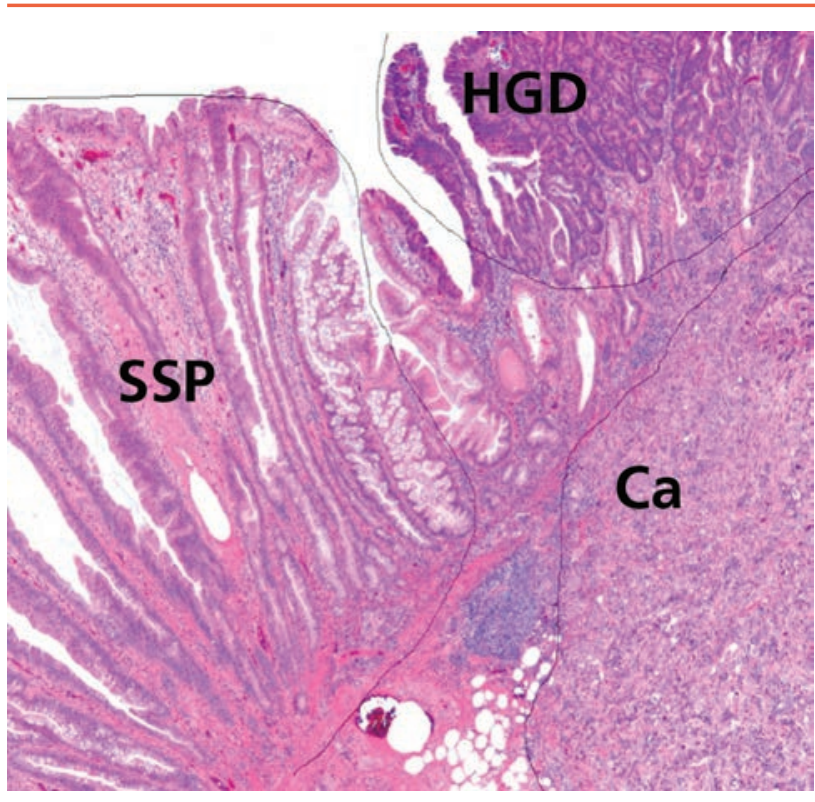

Figure 3 Malignant transformation in the serrated pathway is demonstrated by this polyp that is "caught in the act," with portions showing a sessile serrated adenoma/polyp (SSP) and adjacent areas of cytologic high-grade dysplasia (HGD) and invasive adenocarcinoma (Ca) (hematoxylin-eosin, original magnification $\times 20$ ).

from SSA/P to cancer is approximately 15 years. ${ }^{29}$ Only after longitudinal prospective studies are conducted will the rate of progression and thus clinical risk truly be known.

\section{Clinical Management of SSA/Ps}

\section{Endoscopic Detection}

Colonoscopy and polypectomy are well-known to reduce the incidence of CRC; however, they are less effective at preventing right-sided cancers. ${ }^{41-43} \mathrm{Be}$ cause colon cancers arising via the serrated pathway tend to be right-sided cancers, this is particularly relevant. Perhaps the subtle appearance of SSA/Ps contributes to the lack of recognition or incomplete removal, and the remaining polyps develop into right-sided interval cancers. Thus, it is crucial that endoscopists have a heightened awareness for these lesions and familiarity with their gross appearance.

$\mathrm{SSA} / \mathrm{Ps}$ are usually flat lesions with discrete or indistinct borders, making them difficult to see endoscopically. They can appear as redundant mucosa or a thickened fold with a similar color to the surrounding mucosa. Insufflation during colonoscopy will often flatten the polyp, and thus can make it appear as normal mucosa. The most distinguishing feature of
$\mathrm{SSA} / \mathrm{Ps}$ is the presence of a bronze or yellowish-brown mucous cap, as shown in Figure 4. 2,17,44,45 Tadepalli et $\mathrm{al}^{45}$ designed a review of high-resolution colonoscopic video clips of 158 SSA/Ps, with focus on several gross visual descriptors. The top characteristics that were found to have good-to-excellent intraobserver agreement included the presence of a mucous cap $(63.9 \%)$, rim of debris or bubbles around the lesion $(51.9 \%)$, alteration of the contour of a fold (37.3\%), and interruption of the underlying mucosal vascular pattern $(32.3 \%)$. Another feature recently cited on magnified chromoendoscopic appearance is the recognition of type II open pits, which are thought to represent dilated crypt bases that are wider and rounder than the papillary or stellate (type II) pits found in the HPs and TSAs. This finding was associated with BRAF mutations and CIMP, and is highly specific (97\%) but not sensitive (66\%) for SSA/Ps. ${ }^{46}$ Appreciation of these morphologic characteristics may help endoscopists improve SSA/P detection and thus decrease CRC development.

The difficulty in recognizing these lesions is exemplified by the variability in detection rates. Hetzel et $\mathrm{al}^{26}$ analyzed 4335 polyps from 7192 screening colonoscopies performed by 13 endoscopists. The detection rate per 100 colonoscopies varied from 0.3 to 2.2 for all SSA/Ps, and the prevalence of proximal colon SSA/Ps varied between endoscopists from
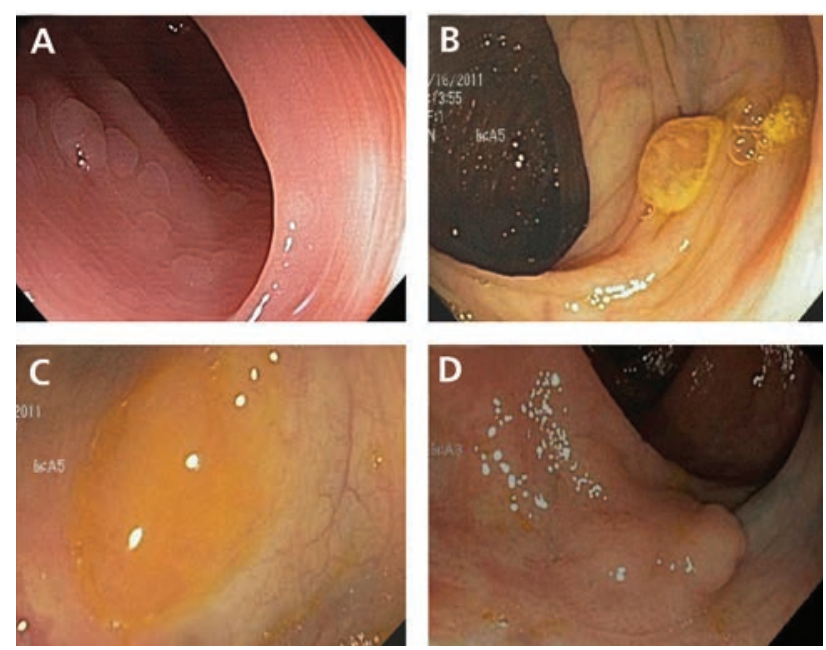

Figure 4 Colonoscopic images of colorectal serrated polyps. Hyperplastic polyps are often small pale lesions that cluster in the rectosigmoid colon (A). Sessile serrated adenomas/polyps (SSA/Ps) can be either small (B) or large (C) and are often covered by a bronze mucous cap as a distinguishing feature. Even large SSA/Ps can be difficult to see, because they tend to be flat and have indistinct borders (D). Courtesy of James Church, MD, Cleveland, $\mathrm{OH}$. 
$0 \%$ to $1.4 \%$. More strikingly, Kahi et $\mathrm{al}^{27}$ reported a detection rate for proximal serrated polyps ranging from $1 \%$ to $26 \%$ among 15 endoscopists. A recent report by Liang et $\mathrm{al}^{47}$ at Cleveland Clinic reviewed 18,003 colonoscopies performed by 6 endoscopists and reported a mean serrated polyp detection rate of $20.6 \% \pm 4.8 \%$ for all examinations, and $13.9 \%$ $\pm 5.0 \%$ for screening examinations only. A regression analysis revealed a significant correlation with adenoma detection rate for screening examinations only. As might be expected, longer withdrawal time was the most important influence on detection rate. Other studies have also suggested a strong correlation between adenoma detection and serrated polyp detection rates. ${ }^{26,27,48}$

Newer technologies, such as narrow band imaging and chromoendoscopy, theoretically may improve detection of serrated lesions. Further studies with particular emphasis on efficacy gained with added time and cost are needed before widespread implementation.

\section{SSA/P Treatment}

Because it is not possible to accurately predict the histology of lesions suspected to be serrated polyps, it is prudent to remove all polyps completely. The exception includes small lesions in the rectum and sigmoid colon that look like hyperplastic polyps. If only a few small polyps are present, then they can be easily removed technically and efficiently. If multiple or clusters of polyps are present that appear to be hyperplastic polyps, then representative biopsies should be performed, but all do not need to be removed.

The general principles of endoscopic removal of SSA/Ps are similar to those used for adenomas, and consideration should be given to size, morphology, and location of the polyp, and to the age and comorbidities of the patient. SSA/Ps tend to be flat lesions and are thus more challenging to remove than pedunculated polyps. However, because SSA/Ps tend to not have submucosal fixation, as is seen with adenomas, the tissue pulls into the snare more easily than flat adenomas, and therefore may actually be easier to remove. Defining the borders of the polyp can often be difficult, and it is important to err on the side of more tissue at the edges. This difficulty is illustrated by a study analyzing the results of endoscopic removal of nonpedunculated polyps that were believed to be completely removed. ${ }^{49}$ For polyps of 5 to $20 \mathrm{~mm}, 10.1 \%$ had positive margins. These finding underscore the difficulty of removing flat polyps, and that incomplete removals may lead to interval cancers.

As with all polyps, removal with a single pass of the snare is preferred to piecemeal resection. If the polyp is believed to be completely removed but was done so in piecemeal fashion, or if any question remains about the completeness of resection, repeat colonoscopy should be performed in 3 to 6 months. Aggressiveness and the extent of removal depend on the experience and judgment of the endoscopist. Elderly patients with comorbidities, bleeding risk, or a thin right colon may preclude aggressive intervention. Furthermore, large flat lesions in the cecum, on the ileocecal valve, or in the appendiceal orifice may not be safely removed because of the anatomy. If a polyp cannot be completely removed, consideration should be given to referral to another endoscopist with more experience with these lesions, or to a colorectal surgeon.

Despite the challenges of removal, large SSA/ Ps can be safely resected in experienced hands. ${ }^{31}$ In a study of 132 large serrated polyps $(\geq 2 \mathrm{~cm})$, Liang et $\mathrm{a}^{31}$ presented a $4 \%$ complication rate, which was statistically similar to that associated with removing adenomas of similar size $(6.9 \% ; P=.376)$. The complications included 3 episodes of bleeding and 1 episode of postpolypectomy syndrome.

\section{Screening and Postpolypectomy Surveillance Recommendations}

As with adenomas, characteristics of serrated polyps influence CRC risk and thus guide surveillance interval recommendations. In general, larger polyps $(\geq 10 \mathrm{~mm})$, polyps located in the proximal colon, and those with dysplasia have increased risk of developing into CRC. In contrast to adenomas, large-scale prospective longitudinal studies on the natural history of serrated polyps are limited, and recommendations have based largely on experience and expert opinion. Current NCCN Clinical Practice Guidelines in Oncology (NCCN Guidelines) for CRC Screening (in this issue) treat SSA/Ps the same as adenomas in terms of personal history and projected CRC risk (to view subsequent updates to these guidelines, visit NCCN.org). ${ }^{50} \mathrm{NCCN}$ recommends that patients with low-risk SSA/Ps $(<1 \mathrm{~cm},<3$ polyps, tubular, no high-grade dysplasia) undergo repeat colonoscopy within 5 years. Advanced (increased 
size, $>25 \%$ villous, or high-grade dysplasia) or multiple SSA/Ps warrant repeat colonoscopy within 3 years. Two expert groups published more detailed recommendations in 2012.,51 The Multi-Society Task Force on Colorectal Cancer presented guidelines for surveillance of all colorectal neoplasia, with an expansion on the topic of serrated polyps in the updated version. ${ }^{51}$ Another group of multispecialty physicians gathered in Cleveland, Ohio for a summit on serrated neoplasia, and produced a review and consensus statement on colorectal serrated neoplasia. ${ }^{2}$ Both groups emphasized the malignant potential of serrated polyps and recommended surveillance and treatment at least as aggressively as for adenomas. A summary of the guidelines are listed in Tables 1 and 2. Both groups admitted that a lack of high level evidence exists on which to base their guidelines, and stated that recommendations will likely evolve as longitudinal studies mature. Recommendations are based on the assumption that colonoscopy was complete and performed after an adequate bowel preparation, and that lesions were completely removed. If a polyp is removed piecemeal, particularly for large, flat, right-sided lesions, a repeat colonoscopy should be performed in 3 to 6 months to assure complete removal. The consensus panel provides a range of intervals for more-advanced SSA/Ps, because of the concern that larger SSA/Ps and SSA/ Ps with cytologic dysplasia may progress quickly into adenocarcinoma. ${ }^{2,38,52}$ Surveillance intervals must be based on individual patient factors within a clinical

\begin{tabular}{|lll|}
\hline Table 1 & $\begin{array}{l}\text { Guidelines for Serrated Polyps by } \\
\text { the US Multi-Society Task Force on } \\
\text { Colorectal Cancer }\end{array}$ \\
Type of Polyp & Size & $\begin{array}{l}\text { Surveillance } \\
\text { Interval (y) }\end{array}$ \\
\hline $\begin{array}{l}\text { Hyperplastic polyps in } \\
\text { rectum or sigmoid }\end{array}$ & $<10 \mathrm{~mm}$ & 10 \\
SSA/Ps & $<10 \mathrm{~mm}$ & 5 \\
SSA/Ps & $>10 \mathrm{~mm}$ & 3 \\
SSA/Ps with dysplasia & Any & 3 \\
TSA & Any & 3 \\
SPS & NA & 1 \\
\hline
\end{tabular}

Abbreviations: NA, not applicable; SPS, serrated polyposis syndrome; SSA/Ps, sessile serrated adenomas/polyps; TSA, traditional serrated adenoma.

Data from Jass JR. Serrated adenoma of the colorectum and the DNA-methylator phenotype. Nat Clin Pract Oncol 2005;2:398-405. context, including patient age, personal and family history of colorectal polyps and cancer, medical comorbidities, and the success of the intervention at that colonoscopy.

The present author believes it is better to err on the side of conservative approach; that is, perform endoscopy more regularly until more information is obtained. Given that the process underlying mucosal changes is often a global field defect, multiple examinations over time provide information regarding the overall stability of the colonic mucosa. If increasing numbers of polyps are present or if there is a rapid size increase in polyp size over a short interval, the author would be inclined to become more aggressive with treatment options or have shorter surveillance intervals. Only after long-term natural history studies are conducted and better-quality evidence is accumulated will the risk be able to be more accurately defined. Future adaptations will be made as further studies are completed.

\section{Serrated Polyposis Syndrome}

Just as adenomas are the hallmark of familial adenomatous polyposis, serrated polyps constitute serrated polyposis syndrome. Formerly called hyperplastic polyposis syndrome because all serrated lesions were classified as HPs, the disease was recently named serrated polyposis syndrome (SPS) by the WHO. ${ }^{1}$ The change in nomenclature reflects the variety of serrated lesions seen in this syndrome, and also stresses the potential premalignant nature of certain serrated lesions. SPS is a clinical diagnosis based on WHO criteria, which are as follows:

- More than 20 serrated polyps of any size, distributed throughout the colon

- At least 5 serrated polyps proximal to the sigmoid colon, with 2 or more of these being larger than $10 \mathrm{~mm}$

- Any number of serrated polyps proximal to the sigmoid colon in an individual who has a firstdegree relative with SPS

SPS prevalence in the general population is difficult to determine because there is an overall lack of familiarity with the syndrome and a lack of recognition. ${ }^{53}$ Of those with recognized SPS, the CRC risk varies greatly depending on the series, but it is likely to be approximately $25 \% .^{2}$ Patients are inclined to 
Kalady

\begin{tabular}{|c|c|c|c|c|}
\hline Histology & Size $(\mathrm{mm})$ & Location of Polyps & Number of Polyps & Surveillance Interval (y) \\
\hline $\mathrm{HP}$ & $<10$ & Rectosigmoid & Any & 10 \\
\hline $\mathrm{HP}$ & $\leq 5$ & Proximal to sigmoid & $\leq 3$ & 10 \\
\hline $\mathrm{HP}$ & Any & Proximal to sigmoid & $\geq 4$ & 5 \\
\hline $\mathrm{HP}$ & $>5$ & Proximal to sigmoid & $\geq 1$ & 5 \\
\hline SSA/P or TSA & $<10$ & Any & $<3$ & 5 \\
\hline SSA/P or TSA & $\geq 10$ & Any & 1 & 3 \\
\hline SSA/P or TSA & $<10$ & Any & $\geq 3$ & 3 \\
\hline SSA/P & $\geq 10$ & Any & $\geq 2$ & $1-3$ \\
\hline SSA/P with dysplasia & Any & & Any & $1-3$ \\
\hline
\end{tabular}

Abbreviations: HP, hyperplastic polyp; SSP, serrated polyposis syndrome; SSA/P, sessile serrated adenomas/polyp; TSA, traditional serrated adenoma. Modified from Rex DK, Ahnen DJ, Baron JA, et al. Serrated lesions of the colorectum: review and recommendations from an expert panel. Am J Gastroenterol 2012;107:1315-1329; with permission.

have synchronous cancers at diagnosis, and metachronous CRC develop. In fact, the polyp phenotype may actually manifest after the initial case of CRC. ${ }^{54}$ The cancer risk is similar whether the patient has the large right-sided phenotype or the small multiple polyp phenotype. ${ }^{54,55}$ In a recent study from Spain, patients with the right-sided polyps tended to have a more significant family history of CRC. ${ }^{55}$ Current NCCN Guidelines for CRC Screening (in this issue) recommend that individuals with SPS undergo screening colonoscopy, with polypectomy of all polyps larger than $5 \mathrm{~mm}$ (to view subsequent updates to these guidelines, visit NCCN.org). ${ }^{50}$ Clearing all larger polyps is preferable, but not always possible. Repeat colonoscopy should be performed between 1 and 3 years, depending on polyp number and size, with a shorter interval if polyps are numerous or larger. ${ }^{50}$ Other expert panels recommend annual colonoscopy because of the increased CRC risk. . $^{251,50}$

Although SPS is believed to have a hereditary component, no heritable genetic or molecular defect has yet been identified. The exact familial risk of SPS is unclear, and screening recommendations for family members of an individual with SPS are debated. Nearly half of patients with SPS have a family history of CRC, ${ }^{54,56}$ and one study reported an elevated relative risk of CRC of 5.4 in first-degree relatives of patients with SPS. ${ }^{57}$ For first-degree relatives of patients with SPS, NCCN Guidelines for CRC Screening recommend screening colonoscopy begin at age 40 years, at the same age as the diagnosis for the youngest family member if uncomplicated by cancer, or 10 years younger than the earliest age at diagnosis of CRC in the fam- ily, whichever is earliest. If no polyps are found, then repeat colonoscopy should be performed in 5 years, but more frequently if polyps are found..$^{50}$ If multiple adenomas or proximal SSA/Ps exist, then 1- to 3-year intervals should be considered. $2,51,50$

Although endoscopy is the cornerstone of SPS diagnosis and management, open communication with surgical colleagues is crucial, and patients must realize the increased cancer risk and the possible need for colectomy. Surgical consultation should be obtained in the following situations: development of cytologic high-grade dysplasia, inability to survey colon regularly or to adequately clear the polyp burden, or rapidly changing size or number of polyps at interval screening examinations. Surgical management usually involves total or subtotal colectomy in the medically fit patient. Any remaining colon or rectum after resection must still be surveyed annually.

\section{Conclusions}

The genetic, molecular, and clinical characteristics of the serrated pathway to neoplasia continue to be unraveled, but it is clearly established as a key player in CRC development. Distinct from the adenomato-carcinoma sequence, the serrated pathway is characterized by BRAF mutations, CIMP, and microsatellite instability. SSA/Ps, as the key intermediaries in this pathway, have malignant potential and are likely responsible for a large number of right-sided interval cancers. Endoscopists must be able to recognize and treat these lesions to help reduce CRC 
Sessile Serrated Polyps

risk. Recent clinical practice guidelines recognize larger size, proximal location, and the presence of dysplasia as risk factors for developing malignancy, and thus recommend surveillance intervals based on these characteristics. Continued development of advanced endoscopic detection technology, further fine-tuning of the genetic characteristics defining malignancy, and longitudinal natural history clinical studies will undoubtedly improve future clinical management.

\section{References}

1. Snover D, Ahnen D, Burt R, Odze R. Serrated polyps of the colon and rectum and serrated polyposis. In: Bosman F, Carrneiro F, Hruban R, eds. WHO classification of tumours of the digestive system. Lyon, France: IARC; 2010:160-165.

2. Rex DK, Ahnen DJ, Baron JA, et al. Serrated lesions of the colorectum: review and recommendations from an expert panel. Am J Gastroenterol 2012;107:1315-1329.

3. Longacre TA, Fenoglio-Preiser CM. Mixed hyperplastic adenomatous polyps/serrated adenomas. A distinct form of colorectal neoplasia. Am J Surg Pathol 1990;14:524-537.

4. Torlakovic E, Snover DC. Serrated adenomatous polyposis in humans. Gastroenterology 1996;110:748-755.

5. Torlakovic E, Skovlund E, Snover DC, et al. Morphologic reappraisal of serrated colorectal polyps. Am J Surg Pathol 2003;27:65-81.

6. Rex DK, Ahnen DJ, Baron JA, et al. Serrated lesions of the colorectum: review and recommendations from an expert panel. Am J Gastroenterol 2012;107:1315-1329.

7. Messick CA, Church J, Bennett A, Kalady MF. Serrated polyps: new classifications highlight clinical importance. Colorectal Dis 2012;14:1328-1337.

8. Bustamante-Balen M, Bernet L, Cano R, et al. Assessing the reproducibility of the microscopic diagnosis of sessile serrated adenoma of the colon. Rev Esp Enferm Dig 2009;101:258-264.

9. Wong NA, Hunt LP, Novelli MR, et al. Observer agreement in the diagnosis of serrated polyps of the large bowel. Histopathology 2009;55:63-66.

10. Sanchez JA, Krumroy L, Plummer S, et al. Genetic and epigenetic classifications define clinical phenotypes and determine patient outcomes in colorectal cancer. Br J Surg 2009;96:1196-204.

11. Vogelstein B, Fearon ER, Hamilton SR, et al. Genetic alterations during colorectal-tumor development. N Engl J Med 1988;319:525-532.

12. Jass JR. Classification of colorectal cancer based on correlation of clinical, morphological and molecular features. Histopathology 2007;50:113-130.

13. Kambara T, Simms LA, Whitehall VL, et al. BRAF mutation is associated with DNA methylation in serrated polyps and cancers of the colorectum. Gut 2004;53:1137-1144.

14. McGivern A, Wynter CV, Whitehall VL, et al. Promoter hypermethylation frequency and BRAF mutations distinguish hereditary non-polyposis colon cancer from sporadic MSI-H colon cancer. Fam Cancer 2004;3:101-107.
15. Hawkins NJ, Ward RL. Sporadic colorectal cancers with microsatellite instability and their possible origin in hyperplastic polyps and serrated adenomas. J Natl Cancer Inst 2001;93:13071313.

16. Park SJ, Rashid A, Lee JH, et al. Frequent $\mathrm{CpG}$ island methylation in serrated adenomas of the colorectum. Am J Pathol 2003;162:815-822.

17. Jass JR. Serrated adenoma of the colorectum and the DNAmethylator phenotype. Nat Clin Pract Oncol 2005;2:398-405.

18. Young J, Simms LA, Biden KG, et al. Features of colorectal cancers with high-level microsatellite instability occurring in familial and sporadic settings: parallel pathways of tumorigenesis. Am J Pathol 2001;159:2107-2116.

19. Burnett-Hartman AN, Newcomb PA, Potter JD, et al. Genomic aberrations occurring in subsets of serrated colorectal lesions but not conventional adenomas. Cancer Res 2013;73:2863-2872.

20. Rosty C, Buchanan DD, Walsh MD, et al. Phenotype and polyp landscape in serrated polyposis syndrome: a series of 100 patients from genetics clinics. Am J Surg Pathol 2012;36:876-882.

21. Whitehall VL, Walsh MD, Young J, et al. Methylation of O-6methylguanine DNA methyltransferase characterizes a subset of colorectal cancer with low-level DNA microsatellite instability. Cancer Res 2001;61:827-830.

22. O'Brien MJ, Yang S, Mack C, et al. Comparison of microsatellite instability, $\mathrm{CpG}$ island methylation phenotype, BRAF and KRAS status in serrated polyps and traditional adenomas indicates separate pathways to distinct colorectal carcinoma end points. Am J Surg Pathol 2006;30:1491-1501.

23. Yang S, Farraye FA, Mack C, et al. BRAF and KRAS mutations in hyperplastic polyps and serrated adenomas of the colorectum: relationship to histology and $\mathrm{CpG}$ island methylation status. Am J Surg Pathol 2004;28:1452-1459.

24. Johannsen LG, Momsen O, Jacobsen NO. Polyps of the large intestine in Aarhus, Denmark. an autopsy study. Scand J Gastroenterol 1989;24:799-806.

25. Williams AR, Balasooriya BA, Day DW. Polyps and cancer of the large bowel: a necropsy study in Liverpool. Gut 1982;23:835-842.

26. Hetzel JT, Huang CS, Coukos JA, et al. Variation in the detection of serrated polyps in an average risk colorectal cancer screening cohort. Am J Gastroenterol 2010;105:2656-2664.

27. Kahi CJ, Hewett DG, Norton DL, et al. Prevalence and variable detection of proximal colon serrated polyps during screening colonoscopy. Clin Gastroenterol Hepatol 2011;9:42-46.

28. Carr NJ, Mahajan H, Tan KL, et al. Serrated and non-serrated polyps of the colorectum: their prevalence in an unselected case series and correlation of BRAF mutation analysis with the diagnosis of sessile serrated adenoma. J Clin Pathol 2009;62:516518.

29. Lash RH, Genta RM, Schuler CM. Sessile serrated adenomas: prevalence of dysplasia and carcinoma in 2139 patients. J Clin Pathol 2010;63:681-686.

30. Spring KJ, Zhao ZZ, Karamatic R, et al. High prevalence of sessile serrated adenomas with BRAF mutations: a prospective study of patients undergoing colonoscopy. Gastroenterology 2006;131:1400-1407.

31. Liang J, Kalady MF, Church J. Snaring large serrated polyps. Surg Endosc 2013;27:1622-1627.

32. Lu FI, van Niekerk de W, Owen D, et al. Longitudinal outcome study of sessile serrated adenomas of the colorectum: an increased 
Kalady

risk for subsequent right-sided colorectal carcinoma. Am J Surg Pathol 2010;34:927-934.

33. Pai RK, Hart J, Noffsinger AE. Sessile serrated adenomas strongly predispose to synchronous serrated polyps in non-syndromic patients. Histopathology 2010;56:581-588.

34. Schreiner MA, Weiss DG, Lieberman DA. Proximal and large hyperplastic and nondysplastic serrated polyps detected by colonoscopy are associated with neoplasia. Gastroenterology 2010;139:1497-1502.

35. Vu HT, Lopez R, Bennett A, Burke CA. Individuals with sessile serrated polyps express an aggressive colorectal phenotype. Dis Colon Rectum 2011;54:1216-1223.

36. Rondagh EJ, Masclee AA, Bouwens MW, et al. Endoscopic red flags for the detection of high-risk serrated polyps: an observational study. Endoscopy 2011;43:1052-1058.

37. Hiraoka S, Kato J, Fujiki S, et al. The presence of large serrated polyps increases risk for colorectal cancer. Gastroenterology 2010;139:1503-1510.

38. Goldstein NS, Bhanot P, Odish E, Hunter S. Hyperplasticlike colon polyps that preceded microsatellite-unstable adenocarcinomas. Am J Clin Pathol 2003;119:778-796.

39. Sawhney MS, Farrar WD, Gudiseva S, et al. Microsatellite instability in interval colon cancers. Gastroenterology 2006;131:1700-1705.

40. Lazarus R, Junttila OE, Karttunen TJ, Makinen MJ. The risk of metachronous neoplasia in patients with serrated adenoma. Am J Clin Pathol 2005;123:349-359.

41. Baxter N, Rabeneck L. New findings about the risks and limitations of colonoscopy used in the early detection of colorectal cancer. Healthc Q 2009;12:24-25.

42. Brenner $\mathrm{H}$, Hoffmeister $\mathrm{M}$, Arndt $\mathrm{V}$, et al. Protection from right- and left-sided colorectal neoplasms after colonoscopy: population-based study. J Natl Cancer Inst 2010;102:89-95.

43. Singh $H$, Nugent Z, Demers AA, Bernstein CN. Rate and predictors of early/missed colorectal cancers after colonoscopy in manitoba: a population-based study. Am J Gastroenterol 2010;105:2588-2596.

44. Rex DK, Hewett DG, Snover DC. Editorial: detection targets for colonoscopy: from variable detection to validation. Am J Gastroenterol 2010;105:2665-2669.

45. Tadepalli US, Feihel D, Miller KM, et al. A morphologic analysis of sessile serrated polyps observed during routine colonoscopy (with video). Gastrointest Endosc 2011;74:1360-1368.
46. Kimura T, Yamamoto E, Yamano HO, et al. A novel pit pattern identifies the precursor of colorectal cancer derived from sessile serrated adenoma. Am J Gastroenterol 2012;107:460-469.

47. Liang J, Kalady MF, Appau K, Church J. Serrated polyp detection rate during screening colonoscopy. Colorectal Dis 2012;14:13231327.

48. Chen SC, Rex DK. Variable detection of nonadenomatous polyps by individual endoscopists at colonoscopy and correlation with adenoma detection. J Clin Gastroenterol 2008;42:704-707.

49. Pohl H, Srivastava A, Bensen $\mathrm{S}$, et al. Incomplete polyp resection during colonoscopy-results of the complete adenoma resection (CARE) study. Gastroenterology 2013;144:74.

50. Burt RW, Cannon JA, David DS, et al. NCCN Clinical Practice Guidelines in Oncology: Colorectal Cancer Screening. Version 2, 2013. Available at: NCCN.org. Accessed November 8, 2013.

51. Lieberman DA, Rex DK, Winawer SJ, et al. Guidelines for colonoscopy surveillance after screening and polypectomy: a consensus update by the US multi-society task force on colorectal cancer. Gastroenterology 2012;143:844-857.

52. Sheridan TB, Fenton H, Lewin MR, et al. Sessile serrated adenomas with low- and high-grade dysplasia and early carcinomas: an immunohistochemical study of serrated lesions "caught in the act". Am J Clin Pathol 2006;126:564-571.

53. Vemulapalli KC, Rex DK. Failure to recognize serrated polyposis syndrome in a cohort with large sessile colorectal polyps. Gastrointest Endosc 2012;75:1206-1210.

54. Kalady MF, Jarrar A, Leach B, et al. Defining phenotypes and cancer risk in hyperplastic polyposis syndrome. Dis Colon Rectum 2011;54:164-170.

55. Guarinos C, Sanchez-Fortun C, Rodriguez-Soler M, et al. Clinical subtypes and molecular characteristics of serrated polyposis syndrome. Clin Gastroenterol Hepatol 2013;11:705-711; quiz e46.

56. Chow E, Lipton L, Lynch E, et al. Hyperplastic polyposis syndrome: phenotypic presentations and the role of MBD4 and MYH. Gastroenterology 2006;131:30-39.

57. Boparai KS, Reitsma JB, Lemmens V, et al. Increased colorectal cancer risk in first-degree relatives of patients with hyperplastic polyposis syndrome. Gut 2010;59:1222-1225. 